\title{
The Penna Model for Biological Ageing on a Lattice: Spatial Consequences of Child-Care
}

\author{
A.O. Sousa and S. Moss de Oliveira \\ Instituto de Física, Universidade Federal Fluminense \\ Av. Litorânea s/n, Boa Viagem, Niterói 24210-340, RJ, Brazil
}

PACS numbers: 87.10.+e, 07.05.Tp, 02.70.Lq

\begin{abstract}
We introduce a square lattice into the Penna bit-string model for biological ageing and study the evolution of the spatial distribution of the population considering different strategies of child-care. Two of the strategies are related to the movements of a whole family on the lattice: in one case the mother cannot move if she has any child younger than a given age, and in the other case if she moves, she brings these young children with her. A stronger condition has also been added to the second case, considering that young children die with a higher probability if their mothers die, this probability decreasing with age. We show that a highly non uniform occupation can be obtained when child-care is considered, even for an uniform initial occupation per site. We also compare the standard survival rate of the model with that obtained when the spacial lattice is considered (without any kind of child-care).
\end{abstract}

\section{Introduction}

The Penna bit-string model for biological ageing was published in 1995 [1], and since then it has been increasingly used to study different characteristics of real populations, as for instance the inheritance of longevity [2] and the advantages of sexual reproduction [3] (for a review, see [4,5] and references therein). Parental care was first introduced in the model by Thoms et al [6]. More recently, it has been shown that different strategies of child-care can lead to smaller or higher effects on the survival probabilities of the population [7], and that when combined with reproduction risk, it leads to a self-organisation of female menopause in sexual populations [8].

In this paper we are mainly interested in two points: the first one is to study the spatial distribution of populations subjected to different strategies 
of child-care. Two of our strategies are specifically related to the movements of the mother on the lattice, and so different from those mentioned above. The third one is also related to the survival of the child, depending if the mother is alive or not $[7,8,9]$. The second point is to compare the usual survival rate of the model with that obtained when the lattice is introduced.

In the next section we describe how the asexual Penna model works on a lattice and our strategies of child-care. In section 3 we present the results considering child-care and in section 4 the results when the standard model is compared with the lattice one (without any child-care strategy). In section 5 we present our conclusions.

\section{The Penna Model on a lattice and strate- gies of child-care}

In the asexual version of the Penna model each individual is represented by a single bitstring of 32 bits, that plays the role of a chronological genome. Each individual can live at most for 32 timesteps ("years"). The first bit gives the information if the individual will suffer from the effects of a genetic disease in its first year of life. The second bit corresponds to the second year of life, and so on. Diseases are represented by bits 1 . Whenever a bit 1 appears it is computed and the total amount of diseases until the current individual's age is compared with a limit $T$. The individual dies when the limit is reached. There is a minimum reproduction age $R$, from which the individual generates with probability 0.5 (in this paper), $b$ offspring every year. We also consider a "pregnancy" period: after giving birth, a mother stays one timestep (year) without reproducing. The offspring genome is a copy of the parent's one, with $M$ random mutations. Only deleterious mutations are considered: if a bit 0 is randomly chosen in the parent's genome, it is mutated to 1 in the offspring one. If a bit 1 is randomly chosen, it stays 1 in the offspring genome.

Besides dying if the number of allowed diseases exceeds $T$ or for reaching 32 years, an individual may also die for lack of space and food. Without a spatial lattice, such lack is taken into account through the general Verhulst factor $V=1-N(t) / N_{\max }$, where $N(t)$ is the actual size of the population at time $t$ and $N_{\max }$ is the maximum size (carrying capacity) the population can sustain, which is defined at the beginning of the simulation [1]. At 
every timestep and for each individual, a random number between 0 and 1 is generated and compared with $V$. If this random number is greater than $V$, the individual dies independently of its age or genome.

In the present case each individual lives in a given site $(\mathrm{i}, \mathrm{j})$ of a square lattice. Instead of considering a single carrying capacity for the whole population, we consider a maximum allowed occupation per site. Moreover, at every timestep each individual has a probability to walk to the neighbouring site that presents the smallest occupation, if this occupation is also smaller or equal to that of the current individual's site. The Verhulst factor is now given by:

$$
V_{i, j}=1-N_{i, j}(t) / N_{i, j(\max )} .
$$

In this way, it is possible to consider the same carrying capacity for all sites, or to define regions with more or less resources. Each individual is first tested: if it does not die because of genetic diseases, lack of food and space (Verhulst) or for reaching 33 years, it moves according to the probability mentioned above. We start the simulations randomly distributing one individual per site on a diluted lattice. That is, if an already occupied site is chosen for a new individual, the choice is disregarded and another random number is generated.

Our strategies of child-care which are purely related to the movements on the lattice consist in defining a period $C_{m}$ during which the mother and the child are forced to stay together: no child can move alone before reaching an age greater than $C_{m}$. In this way, $C_{m}$ is the period during which the child is under maternal care. We have studied the following cases: (a) if the mother moves, she brings all these young children with her; (b) the mother cannot move if she has any child still under maternal care.

We also considered a condition, that can be added to any of the other two or even be considered alone: it consists in killing the motherless child with age $\leq C_{m}$ according to a given probability which decreases with age. We have considered $C_{m}=2$, a probability 0.9 that the child dies in its first year of life if its mother dies, and a probability 0.3 if it happens in its second year of life. This strategy is in fact an improved version of that used before in refs. [7,8], where the offspring die if the mother dies, with probability 1 , at any age inside the child-care period. This improvement is based on data given in ref. [9], fig.5, about baboons and lions. In next section we present our results for cases (a), (b) and (c), where (c) corresponds to case (a) plus 
the survival condition just mentioned.

\section{Results comparing child-care strategies}

In this paper we have worked with the same maximum occupation per site. The figures presented in this section correspond to the following parameters:

I - General parameters of the model

1) Initial population $=10,000$ individuals;

2) Limit number of diseas $T=2$;

3) Carrying capacity (per site) $=34$;

4) Minimum reproduction age $=9$;

5) Birth probability $=0.5$;

6) Birth rate $b=2$;

7) Total number of steps $=800,000$.

II - Parameters related to the lattice and child-care

1) Lattice size $=150$ X 150 sites;

2) Probability to walk $=0.2$;

3) $C_{m}=2 \rightarrow$ age up to which to move alone is forbidden.

With these parameters a maximum population of 160,000 individuals is reached, this number decreasing and stabilizing between 25,000 and 5,000 individuals, depending on the child-care strategy.

In figure 1 we show the final configuration of the lattice for case (a), and in figure 2, for case (b). In figure 3 we present the results for case (c), where two strategies of child care are working together, as mentioned at the end of section 2.

From these figures it can be noticed that the final configuration is strongly dependent on the child-care strategy. Comparing cases (a) and (b), it can be noticed that when the mother cannot move, more empty sites are produced, as a simple consequence of the Verhulst factor: more individuals accumulate at the same site and die for lack of space and food.

When the condition that the child dies if the mother dies is added (fig.3), the final configuration presents more empty sites than fig.1. In this third case 
two effects are superimposed: a stronger action of the Verhulst factor (if the mother is randomly chosen to die, all young children also die), and deaths caused by weak genotypes (mothers that die for accumulation of deleterious mutations). Since the Verhulst factor does not interfere with the genetic features of the population, the survival rates of cases (a) and (b) are the same, and different from that of case (c). In fact, the genetic deaths of mothers with weak genotypes select the best fitted families to survive, increasing the lifespan $[7,8]$, as can be seen from figure 4 . On the other side, the total population decreases the more the stronger is the strategy of child-care considered $[6,7,8]$.

\section{Comparison between the standard results and those obtained with the lattice}

A natural question that appears when the lattice is introduced is: ignoring any child-care strategy, how to compare the standard results of the Penna model with those when the lattice is introduced? Or, which is the behaviour of the survival rate with and without the lattice? In order to answer this question we performed several simulations and observed that:

a) For a probability to walk equal to one, the survival rates of the model with and without the lattice are the same, and no special care must be taken. It means that if one considers, as usual, a given carrying capacity $N_{\max }$ for the whole population, the same survival rate can be obtained simply considering a carrying capacity per site equal to the same $N_{\max }$ divided by the number of sites.

b) As the probability to walk decreases, finite size effects appear. They are observed through a corresponding decrease of the maximum lifetime, that is, the survival rates drop to zero before the standard ones, as can be seen from fig.5a (where the maximum occupation per site is 34 ). In these cases, if one is interested in comparing the lattice results with those of the standard model, a higher occupation per site must be considered. If this occupation is large enough, the results are the same, as shown in fig.5b, where the maximium occupation per site is 200. A maximum occupation per site equal to 100 produces the same result and is so enough to avoid finite size effects.

c) Periodic boundary conditions cannot avoid the finite size effect mentioned 
above, for small occupations per site.

\section{Conclusions}

We show how to implement the Penna model on a lattice, in order to study the spatial evolution of the population. A walking probability is given to each individual, and the Verhulst factor is now per site, i.e., there is a maximum capacity per site. We start the simulations randomly distributing one individual per site, on a diluted lattice. We consider that no individual with age $\leq C_{m}$ can move alone, i.e., while under maternal care. The following strategies of child care have been considered: (a) If the mother moves she brings the offspring under child maternal care with her; (b) The mother cannot move if she has any child under care. Comparing these two situations, we notice that the second one produces more empty sites than the first one, as a consequence of the Verhulst factor (the occupations of many sites exceed the maximum allowed capacity). An extra condition has also been added to the first strategy: if the mother dies, the offspring has a probability to die that depends on its age. In this case, also more empty sites are produced, if compared to the first case. We present the survival rates of the three cases, showing that only the last one interferes with the population genetics.

We also compare the survival rates obtained with the standard model with those obtained when the lattice is introduced (without any kind of child-care). We show that for large enough maximum occupations per site, these survival rates are the same.

Acknowledgements: To P.M.C. de Oliveira, J.S. Sa Martins and A.T. Bernardes for usefull discussions; to CAPES and CNPq for financial support.

\section{References}

[1] T.J.P. Penna, J.Stat.Phys. 78 (1995) 1629.

[2] Paulo Murilo C. de Oliveira, Suzana Moss de Oliveira, Americo T. Bernardes and Dietrich Stauffer, Lancet, 352 (1998) 911.

[3] J.S. Sá Martins and S. Moss de Oliveira, Int.J.Mod.Phys. C9 (1998) 421. 
[4] A.T. Bernardes, Annual Reviews of Computational Physics IV, (1996) 359. Edited by D. Stauffer - World Scientific, Singapore.

[5] S. Moss de Oliveira, Physica A257 (1998) 465; Suzana Moss de Oliveira, Paulo Murilo Castro de Oliveira and Dietrich Stauffer, Evolution, Money, War and Computers, Teubner, Stuttgart-Leipzig (1999).

[6] J. Thoms, P. Donahue, D. Hunter and N. Jan, J.Phys. I5 (1995) 1689.

[7] K.M. Fehsenfeld, J.S. Sá Martins, S. Moss de Oliveira and A.T. Bernardes, Int.J.Mod.Phys. C9 (1998) 935.

[8] S. Moss de Oliveira, A.T. Bernardes and J.S. Sá Martins, Eur.Phys.J. B (1998) in press.

[9] C. Packer, M. Tatar and A. Collins, Nature 392 (1998) 807. 


\section{FIGURE CAPTIONS}

Fig.1 - Final distribution of the population on the lattice considering that if the mother moves, she brings the children under maternal care (age $\leq 2)$ with her (case a).

Fig.2 - Final distribution of the lattice considering that the mother cannot move if she has children under maternal care (case b).

Fig.3 - Final distribution of the lattice considering that if the mother moves she brings the children under maternal care with her, and that if she dies, her children have a probability 0.9 to die in their first year of life, and 0.3 in their second year of life (case c).

Fig.4 - Survival rates for the cases: (a)- filled circles; (b)- triangles; (c)squares.

Fig.5a - Survival rates of the standard model without lattice (triangles), and with lattice for different probabilities to walk. In all cases the initial population $N(0)=10,000$ and the common parameters $(T, b, R$, birth probability) are those described in section 3. In the lattice cases, the maximum occupation per site is 34 and the lattice size is $150 \mathrm{X} 150$. In the standard case $N_{\max }=10 N(0)$.

Fig.5b - Triangles: the same standard survival rate of fig.5a; Full squares: the survival rate with lattice for a maximum occupation per site equal to 200 and a probability to walk equal to 0.2 . 


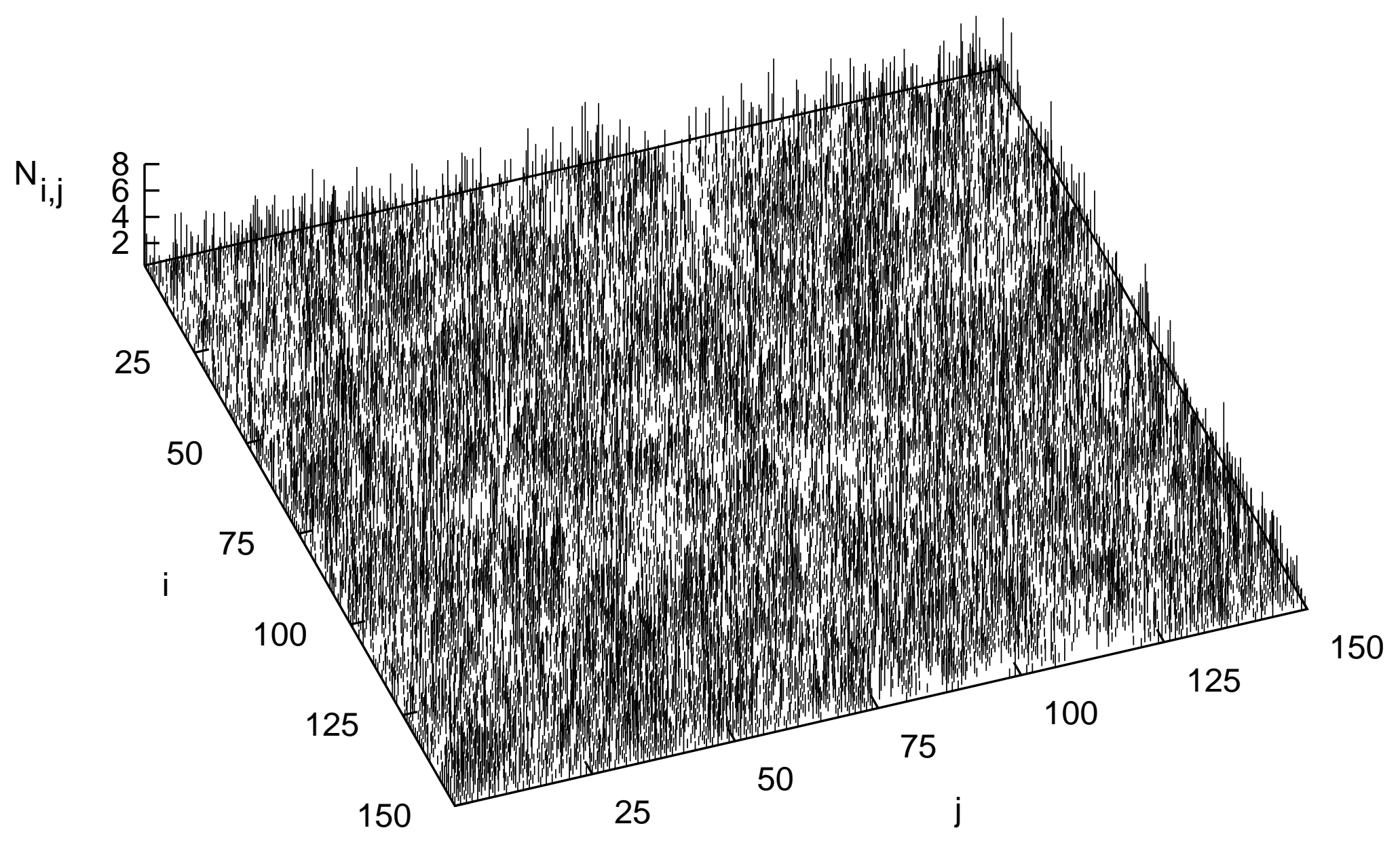




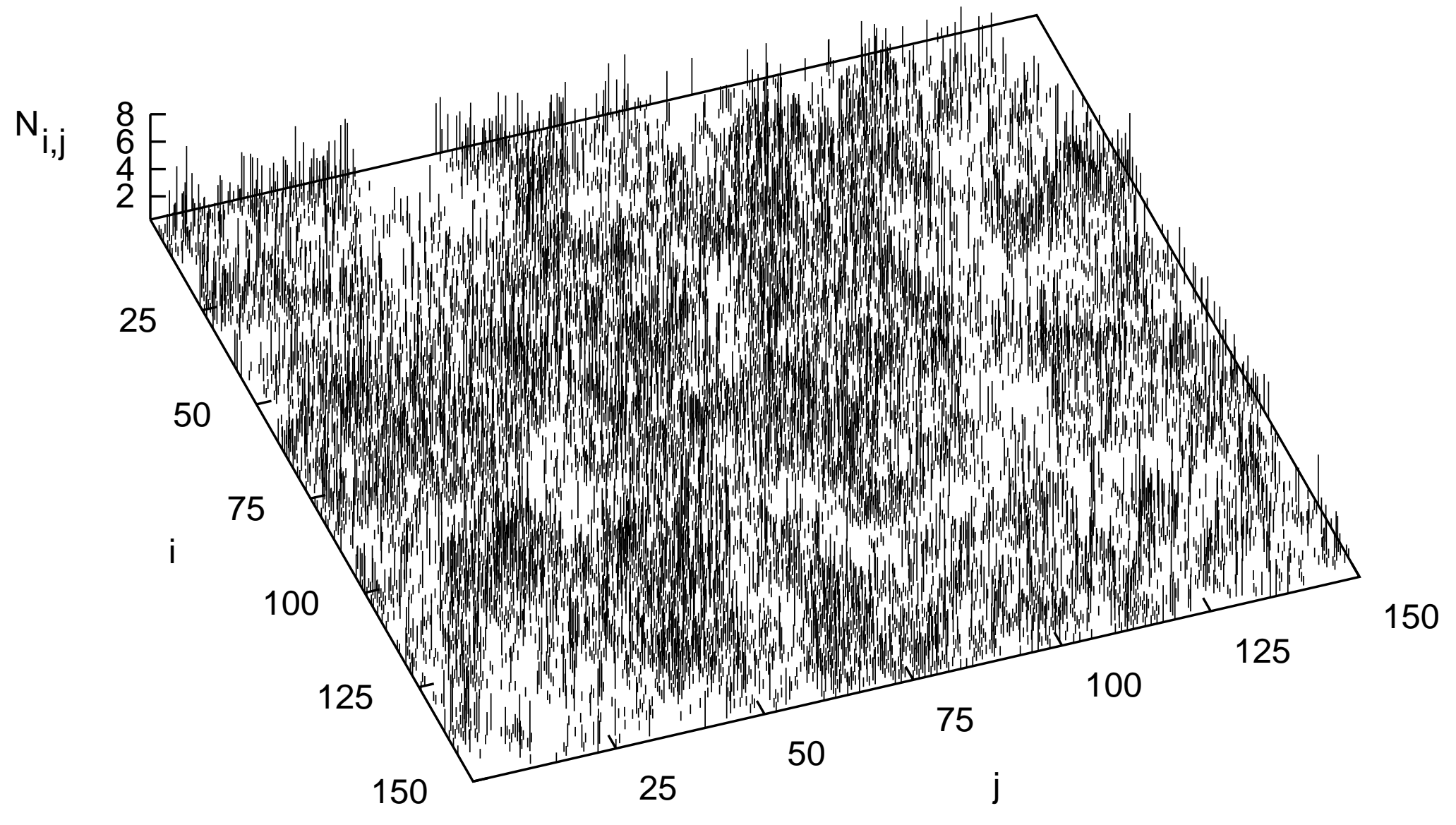




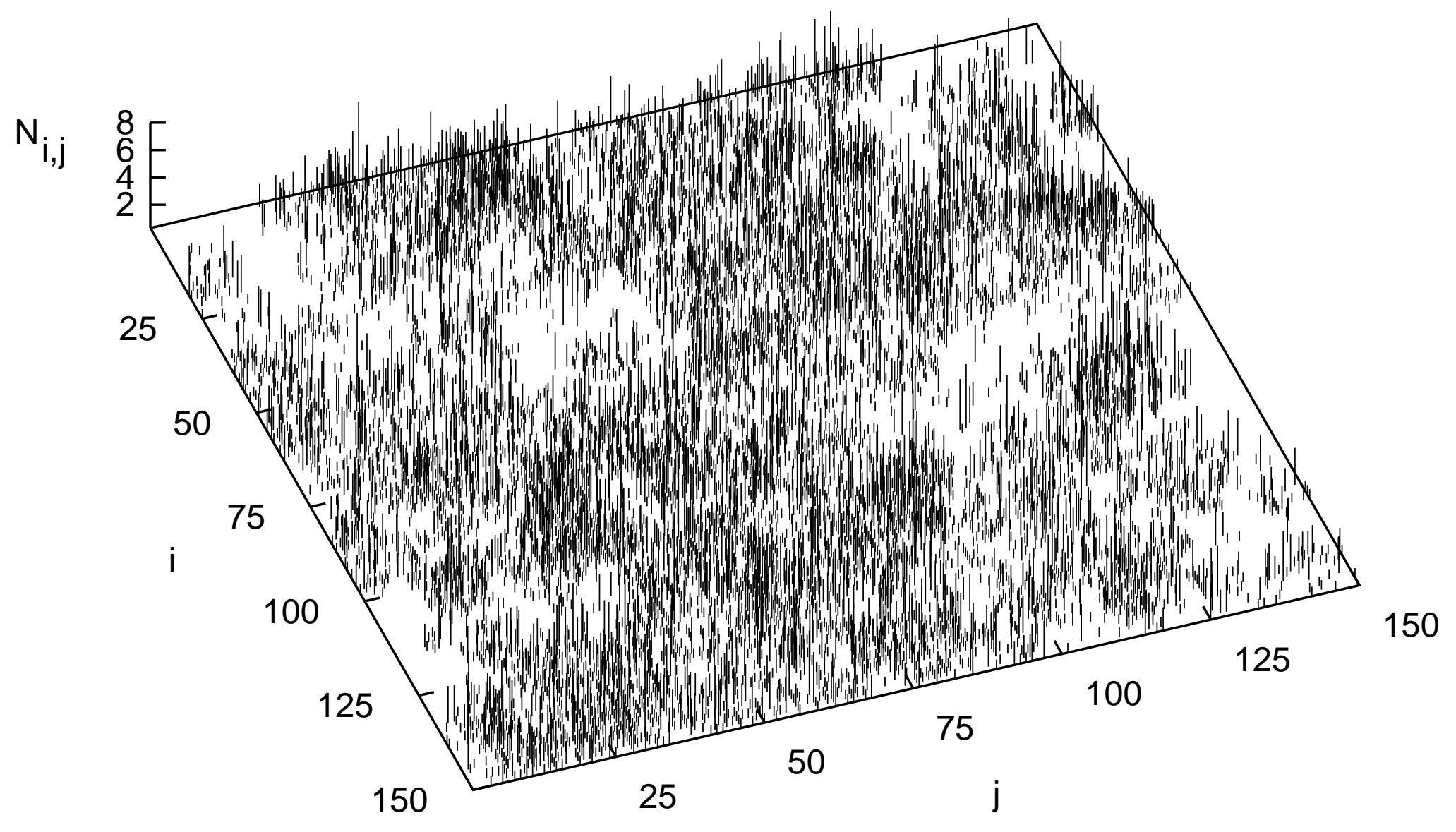




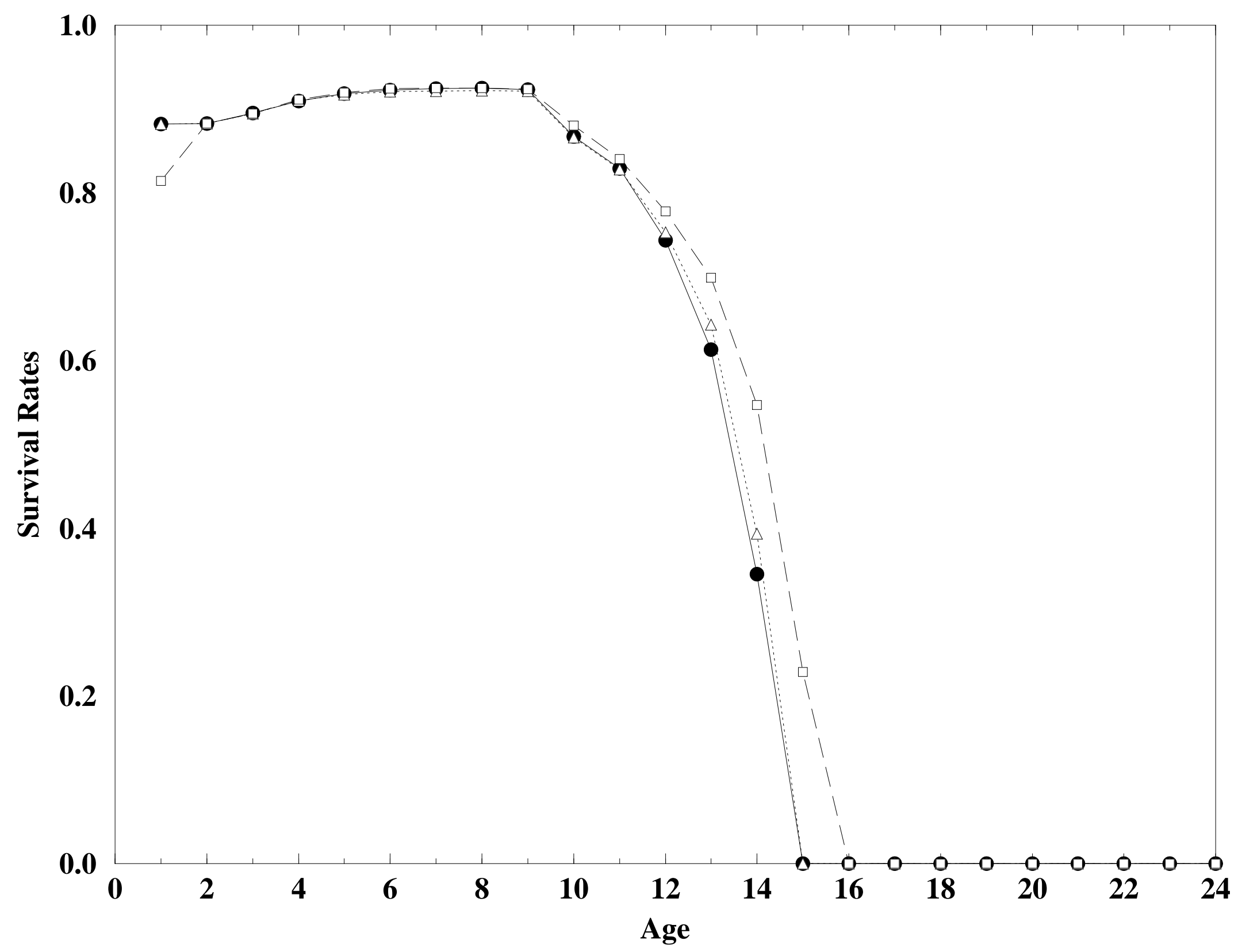




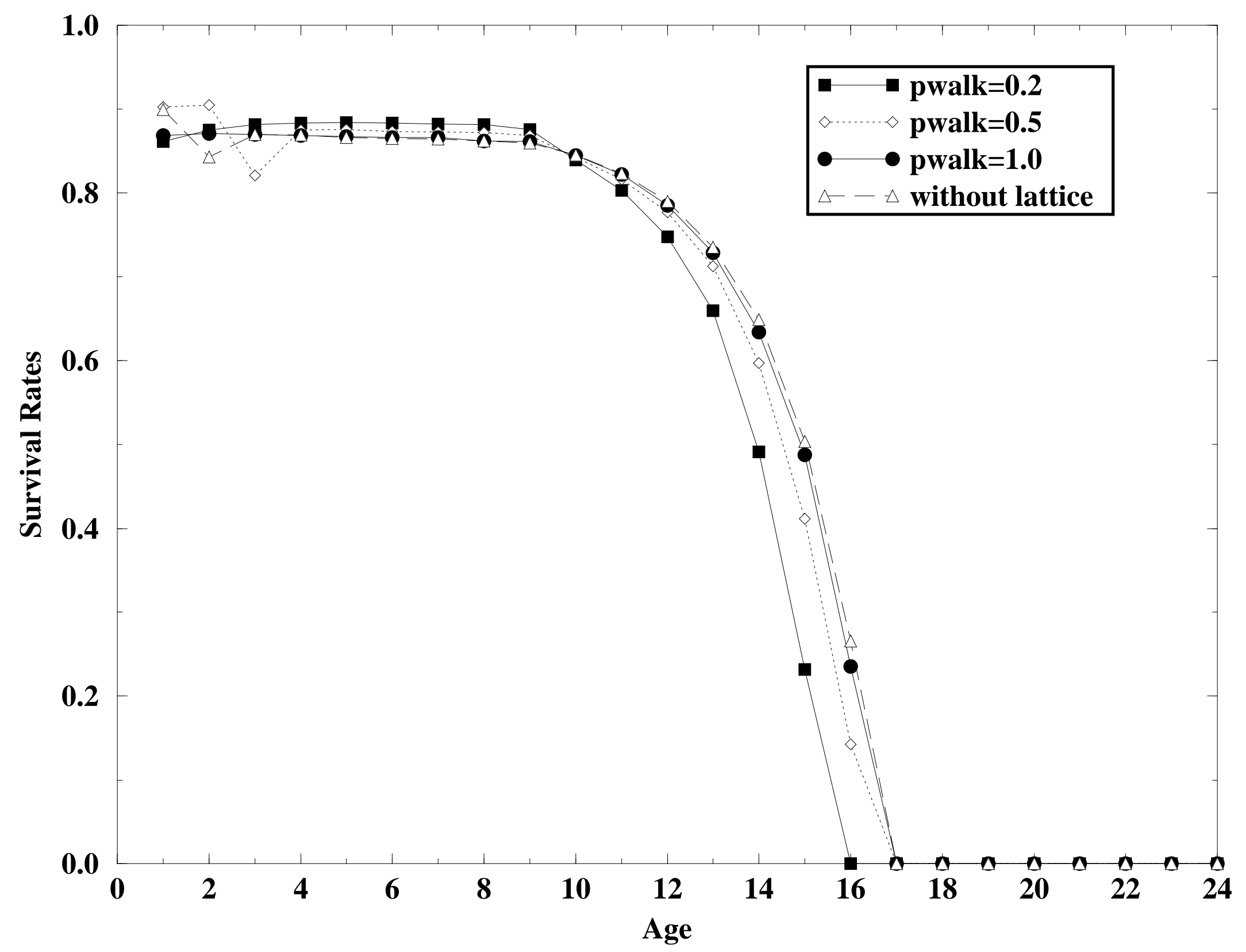




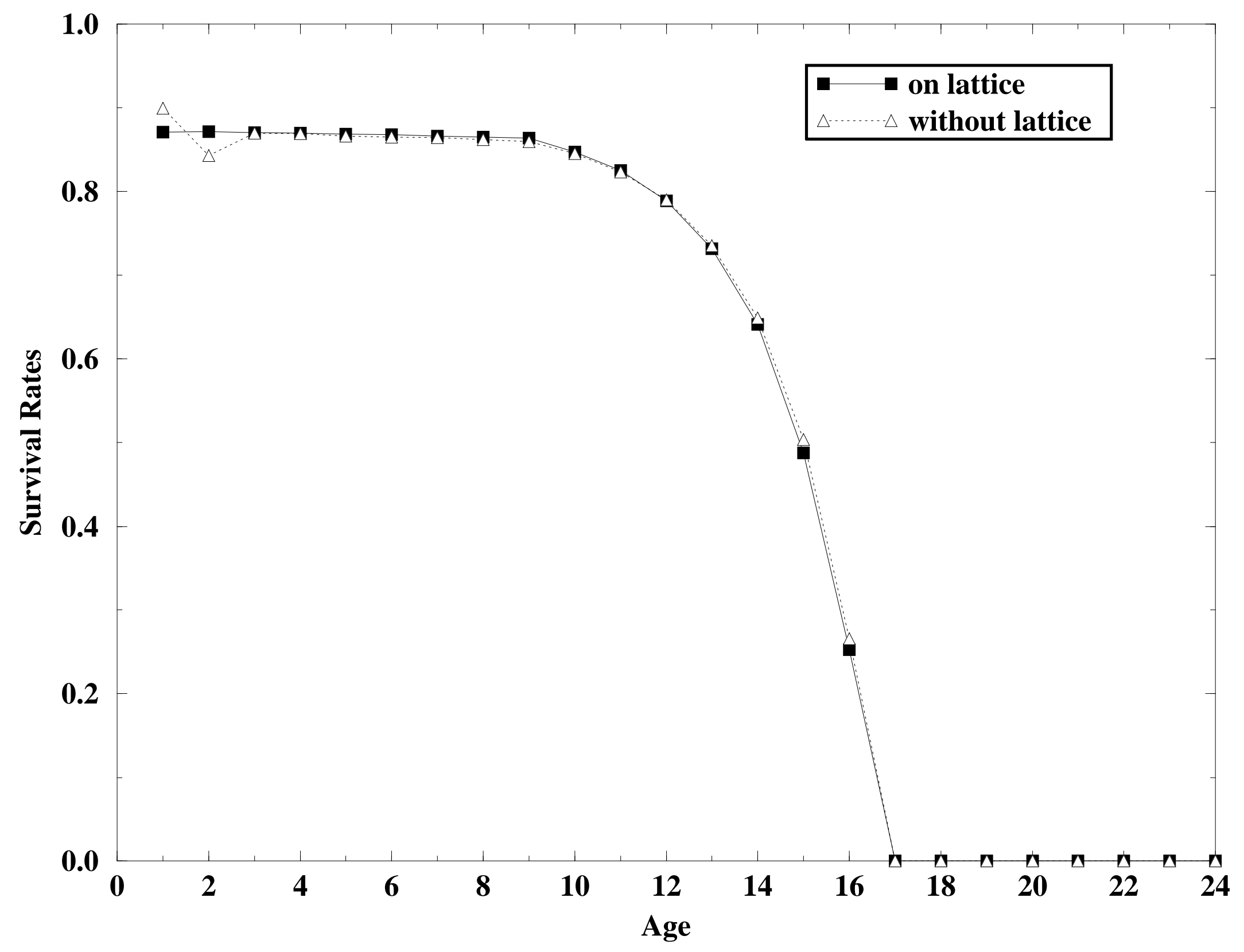

